\title{
CONTEXTOS FORMATIVOS DE LAS PRÁCTICAS DOCENTES PRE- PROFESIONALES EN EL CE.R.P DEL LITORAL
}

\author{
Javier Grilli Silva \\ javier.grilli@gmail.com \\ https://orcid.org/0000-0002-6690-1659 \\ Ce.R.P del Litoral, Dpto. Biología \\ Salto, Uruguay \\ Laura Silva Casterá \\ laucelo@yahoo.com \\ https://orcid.org/0000-0001-8942-8035 \\ Ce.R.P del Litoral, Dpto. Química \\ Salto, Uruguay
}

Recibido: 01/11/2020 Aceptado: 01/12/2020

\begin{abstract}
Resumen
En los meses de abril y agosto del año 2019, se realizó la pasantía en el marco del Proyecto Estudio comparativo de políticas y prácticas institucionales de iniciación e inserción docente, financiado por PASEM. Participaron 4 Universidades de Brasil (Universidad Federal de Ouro Preto Universidad Federal de San Carlos, Universidad Ciudad de San Pablo, Pontificia Universidad Católica de San Pablo) y un Instituto de Formación de Profesores de Uruguay (Centro Regional de Profesores del Litoral). Se pudo constatar en las Universidades participantes, distintos contextos formativos para las prácticas docentes que se desarrollan en la formación inicial de profesores. El Centro Regional de Profesores del Litoral (sede en Salto-Uruguay), en las especialidades de Biología y Química, pone en funcionamiento distintos dispositivos que apuntalan una práctica docente pre-profesional orientada al desarrollo de habilidades reflexivas, cognitivas y metacognitivas, con el objetivo de vincular teoría y práctica en una relación dialéctica. El artículo presenta el contexto formativo que se desarrolla en este Instituto de formación de profesores en Salto, poniendo foco en los procesos de iniciación e inserción a la docencia. Se establecen algunas diferencias con los contextos formativos de las Universidades brasileñas participantes, al tiempo que se presentan algunas fortalezas y debilidades constatadas.

Palabras clave: Formación del profesorado, práctica docente pre-profesional, iniciación a la docencia; inserción a la docencia, práctica docente reflexiva.

\section{CONTEXTOS DE FORMAÇÃO DAS PRÁTICAS DE ENSINO PRÉ-PROFISSIONAL NO CE.R.P DEL LITORAL}

Resumo

Nos meses de abril e agosto do ano de 2019, o estágio foi realizado no âmbito do Estudo Comparativo do Projeto de Políticas e Práticas Institucionais de Iniciação e Inserção de Professores, financiado pelo PASEM. Participaram quatro universidades do Brasil (Universidade Federal de Ouro Preto, Universidade Federal de São Carlos, Cidade Universitária de São Paulo, Pontifícia Universidade Católica de São Paulo) e um Instituto de Formação de Professores do Uruguai (Centro Regional de Professores
\end{abstract}


Costeiros). Foi possível verificar nas universidades participantes, diferentes contextos de treinamento para as práticas de ensino desenvolvidas na formação inicial de professores. O Centro Regional de Professores Costeiros (com sede em Salto-Uruguai), nas especialidades de Biologia e Química, coloca em funcionamento diferentes dispositivos que sustentam uma prática de ensino pré-profissional, voltada ao desenvolvimento de habilidades reflexivas, cognitivas e metacognitivas, com o objetivo de de vincular teoria e prática em um relacionamento dialético. O artigo apresenta o contexto de formação desenvolvido neste Instituto de formação de professores em Salto, com foco nos processos de iniciação e inserção no ensino. Algumas diferenças são estabelecidas com os contextos de treinamento das universidades brasileiras participantes, enquanto são apresentados alguns pontos fortes e fracos confirmados.

Palavras chave: Formação de professores, prática pedagógica pré-profissional, introdução à docência; inserção no ensino, prática pedagógica reflexiva

\title{
TRAINING CONTEXTS OF PRE-PROFESSIONAL TEACHING PRACTICES IN THE CE.R.P DEL LITORAL
}

\begin{abstract}
In the months of April and August 2019, the internship was carrying out within the framework of the Project Comparative Study of Institutional Policies and Practices for Teacher Initiation and Insertion, financed by PASEM. Four Universities from Brazil participated (Federal University of Ouro Preto Federal University of San Carlos, University City of San Pablo, Pontifical Catholic University of San Pablo) and a Teacher Training Institute of Uruguay (Regional Center of Teachers of the Littoral). It was possible to verify in the participating universities, different training contexts for the teaching practices that are developing in the initial teacher training. The Regional Center for Coastal Teachers (based in Salto-Uruguay), in the specialties of Biology and Chemistry, puts into operation different devices that underpin a pre-professional teaching practice oriented to the development of reflective, cognitive and metacognitive skills, with the objective of linking theory and practice in a dialectical relationship. The article explains the training context that is developing in this Institute of teacher training in Salto, focusing on the processes of initiation and insertion into teaching. Some differences are establishing with the training contexts of the participating Brazilian Universities, while some verified strengths and weaknesses are presenting.
\end{abstract}

Key words: Teacher's training, pre-professional teaching practice, initiation in teaching, professional activity initiation, reflective teaching practice.

\section{Introducción}

En el marco del Proyecto presentado al Programa de Apoyo al Sector Educativo del Mercosur (PASEM), denominado Estudio comparativo de políticas y prácticas institucionales de iniciación e inserción docente ${ }^{\mathrm{i}}$, se realizó en los meses de abril y agosto de 2019 el intercambio de experiencias de formación docente entre 4 Universidades de Brasil y un Instituto de formación docente de Uruguay. Las instituciones participantes fueron: Universidad Federal de Ouro Preto (UFOP), Universidad Federal de San Carlos (UFSCar), Universidad Ciudad de San Pablo (UNICID), Pontificia Universidad Católica de San Pablo (PUC-SP) y el Centro Regional de Profesores del Litoral (Ce.R.P del Litoral) perteneciente al Consejo de Formación 
en Educación (CFE) de la Administración Nacional de Educación Pública (ANEP). El objetivo general del Proyecto fue desarrollar un estudio comparativo de políticas y prácticas institucionales de iniciación e inserción docente a partir de diferentes contextos formativos; uno de los objetivos específicos fue compartir estudios, prácticas y experiencias de investigación sobre iniciación e inserción. La iniciación e inserción a la docencia fue por tanto un aspecto de la formación de profesores que tuvo especial atención en las observaciones realizadas a las distintas universidades involucradas en el proyecto.

El Ce.R.P del Litoral es un Instituto terciario no universitario, que forma docentes para la educación media en el interior del país. En su relativa corta existencia (fue creado en el año 1997), ha contribuido de manera significativa a elevar el porcentaje de profesores titulados en el sistema educativo medio de la región litoral del país. Desde su fundación hasta el presente la formación de profesores se dio a través de 3 planes de estudio distintos, conocidos por sus años de aprobación y comienzo: Plan 1997, 2005 y 2008 (Autor, 2020). Cada uno de ellos presenta importantes diferencias en su estructura académica y curricular. En este escrito presentamos experiencias educativas en la práctica docente pre-profesional del actual plan de estudios, Plan $2008^{1}$, desarrolladas en el Ce.R.P del Litoral. Se plantea lo que sucede en 2 especialidades de profesorado que se cursan en el Instituto: Biología y Química.

Uruguay tiene una larga tradición en lo que respecta al peso e importancia dados en la formación inicial de profesores, a las instancias de didáctica y de práctica docente (Pesce, 2014; Rodríguez Zidán, Grilli Silva y Rodríguez, 2020). Con relación a estas prácticas, Branda (2015) platea 4 grandes técnicas para "pavimentar" el camino hacia el desarrollo de un profesional de la docencia: la observación de clases, las micro-experiencias de enseñanza, prácticas preprofesionales y los grupos de reflexión. En el Plan 2008 las 4 están presentes en la propia estructura curricular de las asignaturas de Didáctica correspondientes a los 3 últimos años de la carrera: Didáctica I ( $2^{\circ}$ año), Didáctica II (3er año) y Didáctica III ( $4^{\circ}$ año). En ellas los docentes de las especialidades de Biología y Química del Ce.R.P del Litoral hemos implementados diferentes dispositivos apuntando al desarrollo de un profesional reflexivo y de la mano de ello, un aprendiz colaborativo. Presentamos y analizamos las mismas a la luz de lo que ellas representan para el inicio y la inserción a la docencia.

\footnotetext{
${ }^{1}$ Sistema Único Nacional de Formación Docente 2008 http://www.cfe.edu.uy/images/stories/pdfs/plan_nacional/sundf 2008.pdf
} 
Este artículo presenta algunas características de la práctica pre-profesional docente en el Ce.R.P del Litoral, analizadas en el marco del objetivo del proyecto. El intercambio tenido con los colegas de Brasil nos permitió observar algunas diferencias importantes en las prácticas de aula que los profesores en formación tienen en uno y otro país. El escrito pondrá foco en cómo se da la iniciación e inserción a la docencia en nuestro instituto y en 2 especialidades de profesorado, Biología y Química, estableciendo algunas consideraciones y reflexiones con lo que ocurre en las universidades brasileñas participantes del intercambio.

\section{Iniciación e inserción a la docencia}

Empecemos recordando que la calidad de los docentes y la calidad de la enseñanza que los mismos realizan, tiene una relación muy fuerte con los resultados educativos (CochranSmith y Fries, 2005; OCDE, 2005; Marcelo García, 2008). A su vez la calidad de los docentes guarda también una relación estrecha con su formación profesional, tanto la inicial como la permanente (McKinsey, 2007; Vaillant, 2009; Hargreaves y Fullan, 2014; Vaillant, 2013; Vaillant y Marcelo García, 2015). Por último, señalamos otra relación importante que tiene también suficiente evidencia empírica: las experiencias educativas vividas en la formación inicial del profesor, sobre todo en las instancias de práctica docente, inciden en las formas de enseñar que el docente aplicará en el ejercicio de su profesión y, más aún, en la conformación de su perfil profesional (Davini, 1995; Blanco, 1999; Vezub, 2002; Bolívar, 2007).

Dependiendo de las características que la práctica pre-profesional adopta o tiene en la currícula de la formación inicial, puede representar el inicio a la docencia (Terigi, 2007; Zabalza, 2009), y también una etapa importante en la inserción a la vida profesional docente.

Los términos iniciación a la docencia e inserción a la docencia tienen en ocasiones, un uso indistinto. Si bien los 2 términos implican conceptos muy relacionados, son pasibles de ser diferenciados como lo veremos a continuación. Por otra parte, ambos términos están estrechamente vinculados con un tercer concepto: el de profesor principiante (también llamado profesor novel). Alguien que se inicia en la docencia haciendo prácticas de enseñanza en contextos reales durante la formación de grado, es un profesor principiante; también lo es el egresado de formación docente que se inserta en el mercado laboral. Tanto el que se inicia a la

docencia durante la formación inicial como el que se inserta en el colectivo de profesionales de la educación, son profesores principiantes. El novel profesor está en el proceso de identificar lo 
que implica entrar al sistema educativo y a la organización escolar, donde hay una cultura profesional y donde se pondrán en juego no solo sus habilidades profesionales (conocimiento disciplinar, didáctico y pedagógico), sino también su propia personalidad (Jordell, 1987).

En un sentido amplio la iniciación a la docencia comienza con la formación de grado para ser educador. Es aquí que el aprendiz docente tiene las primeras experiencias de enseñanza en contextos reales. Diferentes estudios han abordado las diferentes fases del período de iniciación a la docencia, fases que debe ir superando el profesor principiante para poder convertirse en un verdadero profesional de la docencia (Tardif, 2004; Imbernón, 2007). La duración de estas fases es variable, abarcando la formación inicial y también los primeros años de egreso.

Por otra parte, tenemos la inserción a la docencia, llamado también por algunos autores inducción a la docencia. Es la etapa de la carrera docente de aquellos que terminan un programa de formación inicial y empiezan a laborar. En este momento dos dimensiones interactúan de manera simultánea: la búsqueda de empleo y el inicio de la carrera docente (Tardif y Borges, 2013). La inserción a la docencia puede durar varios años y "es el momento en que el maestro novato tiene que desarrollar su identidad como docente y asumir un rol concreto dentro del contexto de una escuela específica." (Vaillant, 2009, p. 35). Se caracteriza esta etapa por las tensiones y aprendizajes intensivos, donde el profesor principiante debe adquirir conocimiento profesional y conseguir mantener cierto equilibrio personal (Vonk, 1996).

Decíamos anteriormente que en la formación de grado se tienen las primeras experiencias de enseñanza en contextos reales: la práctica docente pre-profesional. Ella es la etapa en la que los docentes en formación inicial entran en contacto con su medio profesional, poniéndose de manifiesto la interacción entre la teoría y la práctica (Rodríguez, Calle y Zabala, 2018). Las prácticas de enseñanza que se dan en la formación inicial de los docentes, así como también en los primeros años de ejercicio profesional, son momentos claves en el "proceso de convertirse en docente" (Bullough, 2000; Correa, Cividini y Fuentealba, 2013). Por esto es muy importante que en ellas se establezcan las bases para ser un "profesional reflexivo": enseñante que reflexiona críticamente sobre su práctica, tomando decisiones en cada situación, con cada alumno y con cada grupo (Schön, 1992; Zeichner, 1995; Freire, 1997; Perrenoud, 2004).

Esta cualidad, ser un docente reflexivo, debe ser explicitada y debidamente enseñada en la formación inicial, principalmente en los espacios de didáctica y práctica pre-profesional. Es 
aquí donde "el estudiante debe apropiarse de las habilidades específicas de la actividad profesional, manifestando su lógica de pensar y actuar en condiciones concretas de la profesión y del trabajo.” (Rodríguez, Calle y Zabala, 2018, p. 22). Con esto se apunta también al desarrollo de un docente autónomo, es decir que sabe analizar sus aciertos y sus fallas cuando enseña, para así poder continuar mejorando su praxis.

Como sostiene Elsa Meinardi, la "práctica reflexiva debe darse a través del conocimiento profundo de los contextos en los cuales opera" (Meinardi, Arias y Plaza, 2018, p. 9). Es a partir de actuar y resolver situaciones de enseñanza y aprendizaje en un determinado contexto de práctica pre-profesional, que el profesor en formación va desarrollando competencias para el ejercicio de la docencia. Es en esta práctica docente donde se deben hacer funcionar dispositivos que viabilicen una profunda y constante reflexión teórica y meta-teórica (Grilli Silva y Silva Casterá, 2015; Arias Regalía, Bonan y Gonçalves, 2016). Se trata de reflexionar en la acción y sobre la acción; reflexionar metacognitivamente revisando las concepciones del docente sobre qué es enseñar y qué es aprender, concepciones que además están muy arraigadas en el profesor en formación porque forman parte de su propia biografía escolar (Meinardi, Arias y Plaza, 2018).

Como lo demuestran los estudios de Barth, los maestros principiantes en sus primeros años de desempeño, son aprendices voraces que se preocupan por aprender su nuevo oficio (citado por Day, 2005). Esto es algo que debe ser explotado en las instancias de práctica preprofesional. Estas primeras experiencias de enseñanza deben establecer las bases y crear hábitos profesionales para ser un docente abierto al cambio, a la innovación y a la superación.

\section{La práctica docente en la formación inicial de profesores. Experiencias educativas en el Ce.R.P del Litoral}

En Uruguay, la formación de grado de los Profesores para Educación Media depende de la ANEP y dentro de ella está a cargo un órgano desconcentrado: el CFE. Existen 21 especialidades en la carrera de profesorado que se corresponden con diferentes campos del saber: matemática, ciencias biológicas, química, literatura, informática, inglés, entre otras.

La didáctica y la práctica docente son reconocidas dentro de las bases que dan estructura e identidad a los profesionales de la educación. Como sostiene Pesce (2014), la Didáctica en la 
carrera de profesorado presenta un perfil propio vinculado estrechamente a las disciplinas que se enseñan y a las prácticas de enseñanza implicadas.

Conforme a la estructura curricular del plan actual, Plan 2008, la práctica docente preprofesional del profesor en formación se da conjuntamente con la formación en didáctica. Las asignaturas en las cuales transcurre la práctica docente se denominan: Didáctica I, Didáctica II y Didáctica III, correspondientes respectivamente a $2^{\circ}, 3^{\circ}$ y $4^{\circ}$ año de la carrera docente. Estas asignaturas constituyen unidades formativas entre aspectos teóricos del campo de saber de cada didáctica específica y la práctica profesional que involucra la enseñanza de las diferentes especialidades. El profesor de estas asignaturas es docente de didáctica y tutor de práctica docente; aborda contenidos teóricos de la didáctica y paralelamente realiza el acompañamiento y supervisión de la práctica docente (Grilli Silva y Silva Casterá, 2015).

Los cursos de Didáctica I y II comparten como característica común la existencia de una tríada formativa (docente de didáctica, docente adscriptor y estudiante). Esto se desarrolla en el marco de dos instituciones educativas: el centro de formación en educación y el centro de educación media donde se realiza la práctica. El estudiante asiste a un grupo de educación media que se encuentra a cargo de un docente que cumple requisitos establecidos (puede pertenecer tanto al Consejo de Educación Secundaria como al Consejo de Educación Técnico Profesional) y en ese grupo denominado grupo de práctica realiza observaciones e intervenciones didácticas. La frecuencia y carácter de dichas intervenciones tienen carácter gradual y constituyen procesos con andamiajes aportados por el profesor de didáctica y adscriptor quienes trabajan coordinadamente.

En la figura 1 representamos la organización general de la asignatura Didáctica III para el profesorado de Biología en el Ce.R.P del Litoral. Se tienen en el Instituto 2 encuentros semanales con los estudiantes, de 1 hora y media de duración cada uno. En uno de los 2 días se desarrolla el "curso teórico-práctico de didáctica" que comprende 5 módulos temáticos, titulados: La Programación Didáctica desde una concepción constructivista de la enseñanza y del aprendizaje; Características de la práctica docente en la formación inicial de un profesor; Para qué enseñar Biología en el nivel educativo medio; Estrategias y recurso para enseñar y aprender Biología en los tiempos actuales; Ética de la profesión docente en las acciones de aula. En la tabla 1 se presenta un listado de los principales conceptos que se abordan en cada módulo temático. 
Figura 1. Organización general del curso de Didáctica III en Profesorado de Biología

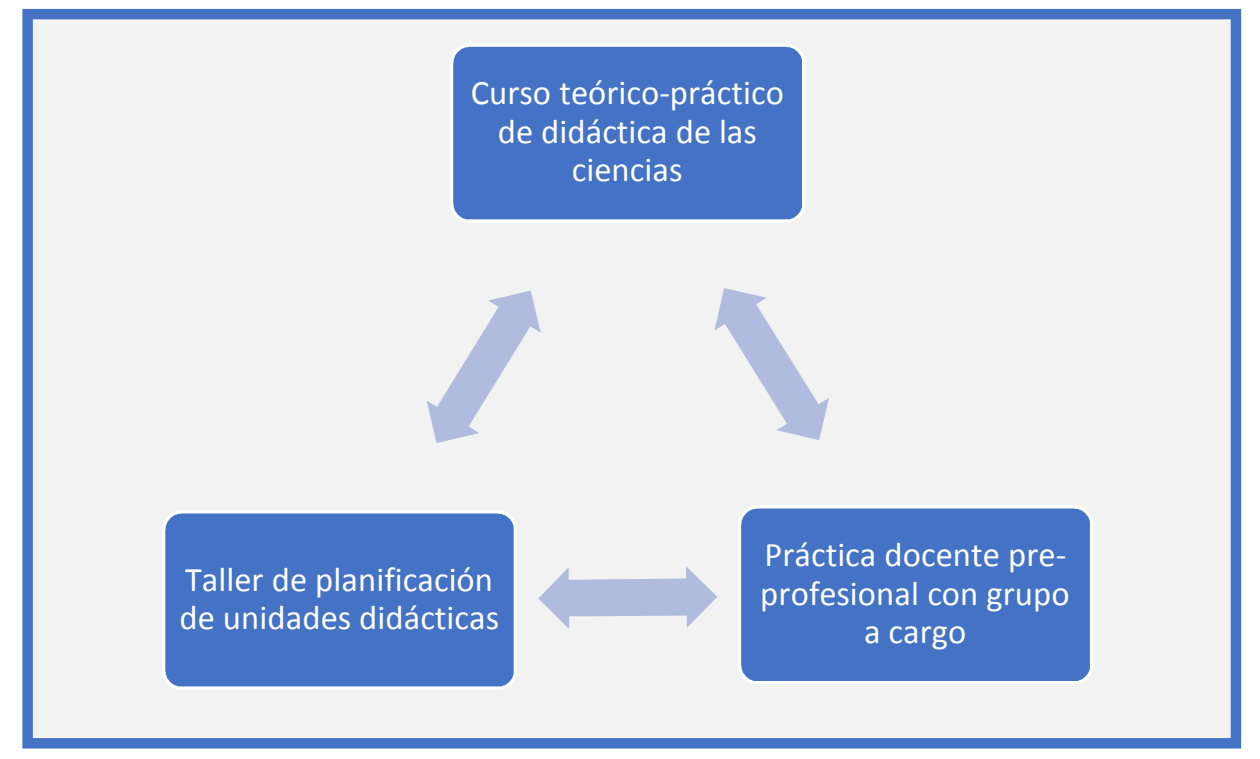

Fuente: elaboración propia

Tabla 1. Curso teórico-práctico de Didáctica III en Profesorado de Biología

\section{Módulo Temático}

1. La Programación Didáctica desde una concepción constructivista de la enseñanza y del aprendizaje
Principales conceptos que se abordan
2. Características de la práctica docente en la formación inicial de un profesor
Programar el primer día de clase

Niveles de concreción curricular

Niveles de Programación Didáctica

Elementos de una Programación de Aula

Gestión del aula

Relevancia de los contenidos procedimentales en la enseñanza de la Biología

Contenidos procedimentales vinculados a: lo lingüístico y la ilustración científica.

Actividades y tareas de la Programación Didáctica.

Evaluación de los aprendizajes. Tipos e instrumentos.

Complejidad, singularidad y simultaneidad de interacciones en la PD

Identidad profesional docente

Modelos, enfoques y perspectivas sobre la PD.

Práctica individual y práctica colectiva; comunidades de aprendizaje.

Auto-confrontación y práctica reflexiva. 
3. Para qué enseñar Biología en el nivel educativo medio

4. Estrategias y recurso para enseñar y aprender Biología en los tiempos actuales
Que Biología enseñar

Ciencia escolar

Educar para la ciudadanía

Educación científica para desarrollar el pensamiento

Educar para la vida

Educar para la cotidianidad

Alfabetización científico-tecnológica

Enfoque CTS

Funcionalidad del aprendizaje

Ciencia y educación en valores.

Aprendizaje basado en problemas.

Medios de Enseñanza

Pizarrón clásico y digitales

Actividades de laboratorio

Educar en una cultura del espectáculo

Cine: comercial, científico y de ciencia ficción

Cine y ciencia en el aula

Ilustración científica

Actividades lúdicas en el aula.

Videojuegos y educación

Publicidad y ciencia

El video-clip musical

El humor en la enseñanza de las ciencias.

Dibujos animados y Biología

TIC y triángulo didáctico

La PC en el aula

Recursos informáticos

Ética y moral

La ética docente en el trabajo de aula

Coordenadas éticas de la profesión docente

El ethos de la profesión docente

Fuente: elaboración propia en base al Programa Oficial de Didáctica III de profesorado de Biología -1 Plan 2008

En el segundo encuentro semanal que tiene la asignatura, se desarrolla el "taller de planificación didáctica" orientado a la práctica pre-profesional. A lo largo del año se van analizando entre profesores practicantes que comparten una misma asignatura de ciclo básico medio, junto con el profesor de didáctica, posibles estrategias y recursos para la enseñanza de los temas propuestos por el programa oficial de la asignatura de nivel educativo medio en la que se hace la práctica pre-profesional. En el taller se trazan entonces los principales lineamientos para la elaboración de las unidades didácticas: se definen objetivos de aprendizajes, contenidos a enseñar, posibles estrategias y recursos a utilizar. La temporalización de las unidades a lo largo del año, así como también de las actividades y tareas de enseñanza para cada una, se discuten grupalmente en los talleres.

Tanto el curso teórico-práctico de didáctica como el taller de planificación, se desarrollan en paralelo a la práctica pre-profesional que el estudiante de $4^{\circ}$ año tiene. El curso 
teórico se nutre de ejemplos emanados de la práctica pre-profesional al tiempo que los autores y conceptos de la didáctica que se enseñan, se van ejemplificando con situaciones reales de la práctica docente que realizan los profesores en formación. Por su parte en los talleres de programación didáctica se toman decisiones, se opta por tal o cual estrategia y recurso, haciéndose explícito el aspecto teórico didáctico (el autor, el concepto), que se utiliza para ello. Se da por tanto una fuerte interrelación entre curso teórico-práctico de didáctica, taller de planificación didáctica y práctica docente pre-profesional (figura 1).

La práctica pre-profesional que se da en $4^{\circ}$ año es, como ya se explicó más arriba, con un grupo a cargo. La tutoría del profesor principiante en su práctica pre-profesional, se da a través del profesor de Didáctica III; las distintas instancias de acompañamiento y de supervisión que se realizan están esquematizadas en la figura 2. Las visitas de aula a las clases del profesor practicante, son la instancia fundamental en la función tutorial. En Biología se realizan habitualmente entre 6 y 7 visitas de aula a lo largo del año, a cada uno de los practicantes (unos 10 o 12 estudiantes en cada cohorte). Generalmente se hace una visita por mes a partir del mes de abril. En la visita el profesor de didáctica no realiza intervenciones en el aula, adoptando un rol de observador. Algunas de las visitas se realizan de manera colectiva: el profesor de didáctica coordina con 2 o 3 practicantes para ir juntos al grupo-clase de un compañero y hacer así una observación conjunta. De esta manera 2 o 3 profesores practicantes acompañan al profesor de didáctica, para observar el desarrollo de la clase. Durante los 45 minutos que dura la clase, se hace circular entre los practicantes observantes, distintos ítems o puntos de observación/reflexión, de lo que está sucediendo.

Figura 2. Instancias para el acompañamiento y supervisión de la Práctica Docente en Didáctica III de Biología

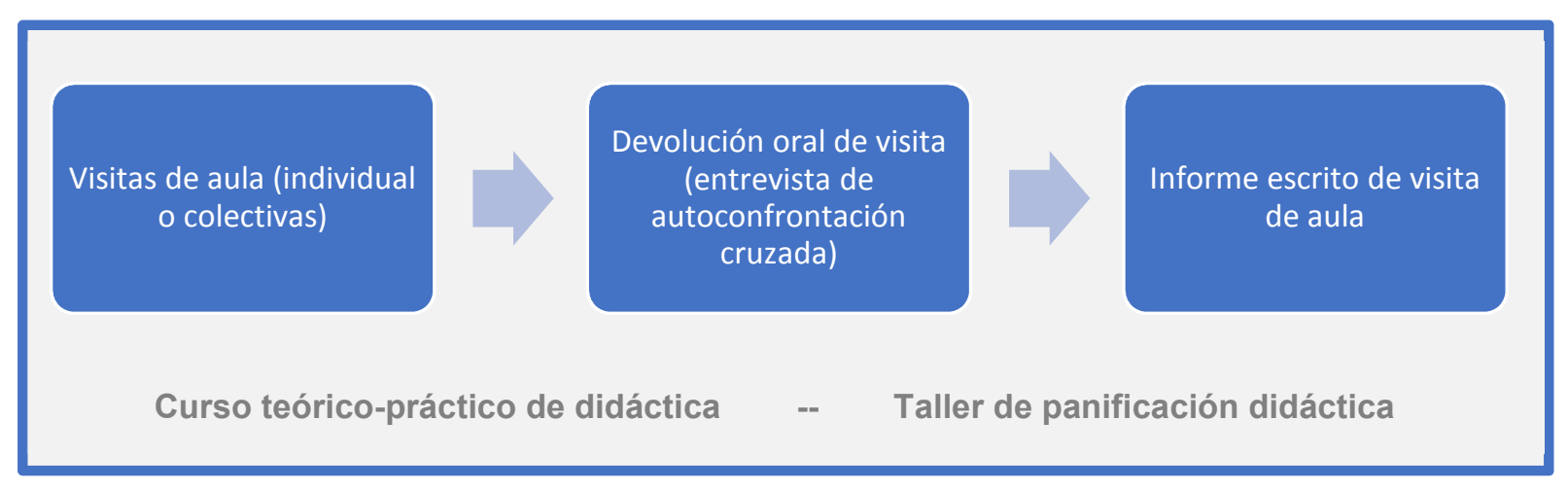

Fuente: elaboración propia 
Las visitas de aula que se hacen a lo largo del año, tanto las individuales como las colectivas, van seguidas de una "devolución de clase". La misma tiene un formato de tipo "entrevista de auto-confrontación cruzada" (Fernández y Clot, 2010; Rickenmann, 2007). Se hacen el mismo día de la visita de aula, o al siguiente. Primero es el propio profesor practicante que condujo la clase observada, quien hace un análisis de su desempeño; luego esto es confrontado por el "espejo" que está representado por las observaciones que hacen los compañeros practicantes y el profesor de didáctica.

Cada visita de aula concluye con un informe escrito realizado por el profesor de didáctica. El formato general del mismo se presenta en la figura 3, y es común a los profesorados de Biología y Química. Se recogen aquí fortalezas y debilidades observadas, que se presentan vinculadas a conceptos y autores de la didáctica estudiados en el curso teórico. También se plantean valoraciones y reflexiones que fueron mencionadas durante la instancia de devolución de visita, tanto aquellas que menciona el propio practicante visitado, como las que hacen sus

Figura 3. Formato general del informe de visita en Didáctica III de Biología y Química

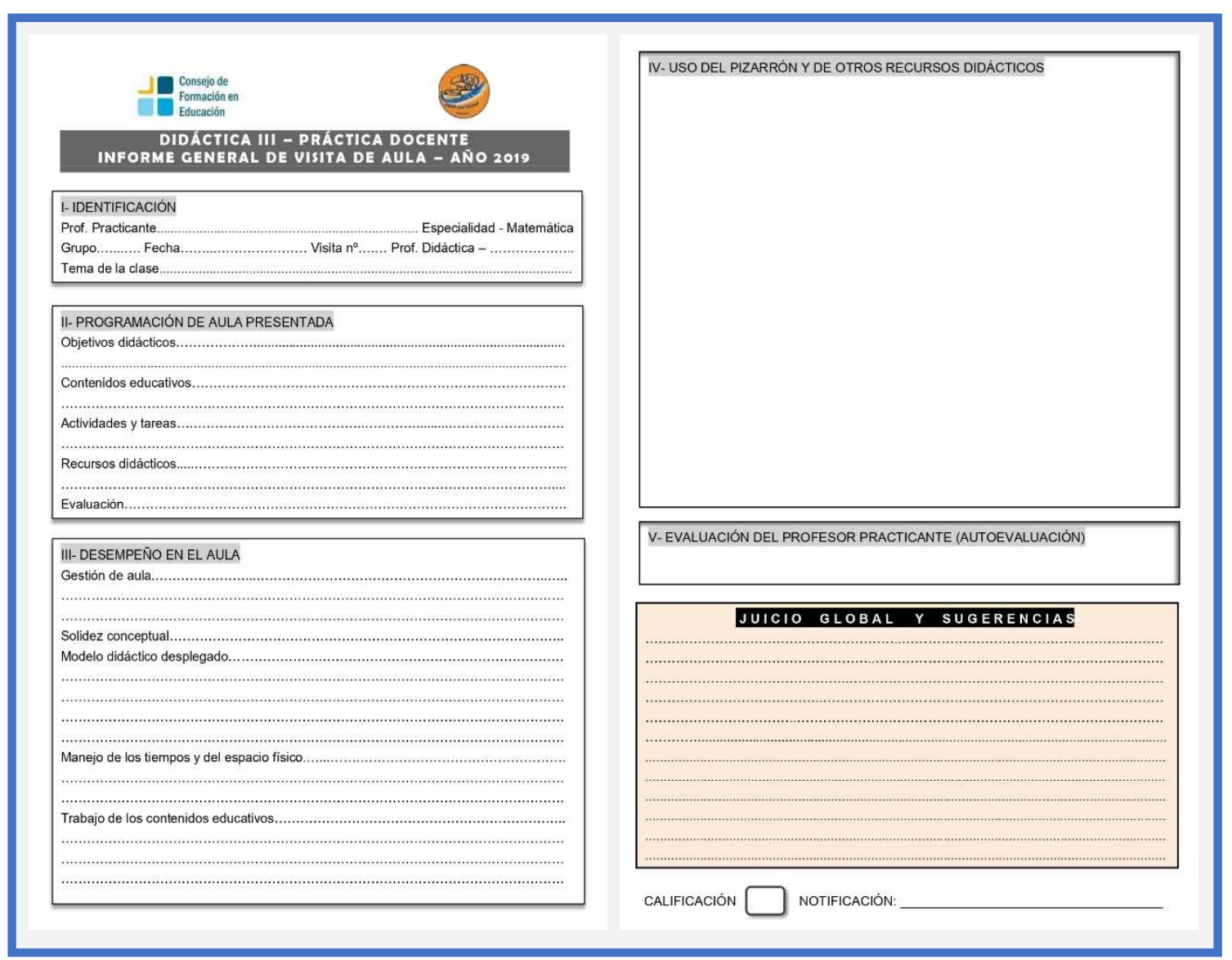

Fuente: elaboración propia 
compañeros visitantes. Todo el proceso de tutoría de la práctica docente pre-profesional, está inmerso en el curso teórico-práctico de didáctica y en los talleres de programación didáctica (figura 2).

Se apunta a una práctica pre-profesional reflexiva donde se produzca una dinámica que sea a la vez personal y colectiva (Rodríguez, Calle y Zabala, 2018). En las diferentes instancias y actividades que se desarrollan en Didáctica III (curso teórico, taller de programación didáctica, visitas individuales, visitas colectivas, devoluciones orales de clase e informes escritos de visitas), afloran concepciones didácticas y pedagógicas, aciertos y errores en la planificación... siendo algunos personales y no compartidos por otros, o comunes a varios (a veces todos los practicantes).

Las instancias y actividades del curso de Didáctica III antes mencionadas, permiten transitar por lo que Calle García, Rodríguez Andino y Amayuela Mora (2015) denominan 3 momentos esenciales del proceso de reflexión en las prácticas pre-profesionales: reflexión colectiva antes de la acción docente, reflexión individual en la acción y reflexión colectiva de la acción realizada. Los talleres de programación didáctica y el curso teórico-práctico de didáctica, son andamios por donde transitar la reflexión colectiva antes de la enseñanza; las visitas de clases y las devoluciones orales de las mismas, viabilizan el proceso de reflexión individual; las devoluciones colectivas, los informes de visitas realizados y los ejemplos de la práctica que se utilizan para desarrollar el curso teórico-práctico, son importantes instancias para la reflexión colectiva de la acción realizada.

Entre los cursos de Didáctica III de las especialidades Biología y Química existen planos formativos comunes: carga horaria semanal del curso teórico, talleres de planificación, vinculación entre curso teórico y práctica pre-profesional, acompañamiento y supervisión de dicha práctica, así como la metodología de trabajo respecto a visitas de aula y su posterior tratamiento didáctico.

\section{Tabla 2. Programa de Didáctica III en Profesorado de Química}

1. La Didáctica y el Planificación anual Niveles de concreción curricular: currículo

-Plan anual, diseño de unidades didácticas, plan diario.

-Evaluación diagnóstica.

-El trabajo en proyectos educativos. 


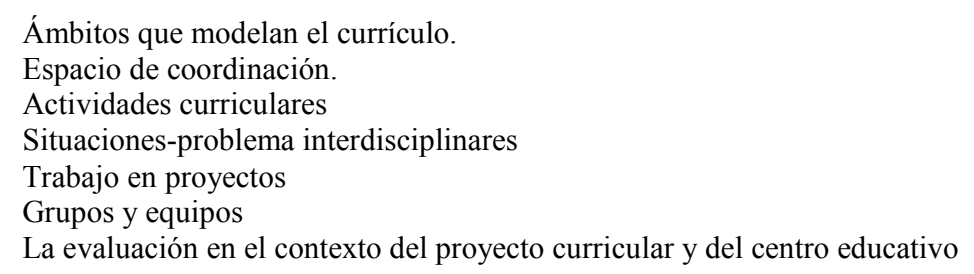

2. La enseñanza y el El aprendizaje como factor motivacional

aprendizaje Construcción de conocimiento. Concepciones de aprendizaje y de enseñanza: Piaget, Vigotsky, Bruner, Ausubel.

Introducción a las propuestas teóricas didácticas desde los aportes de neurociencias (neurodidáctica).

Metacognición:

a-concepciones de metacognición.

b-metacognición y cognición.

c-relaciones de metacognición con aprendizaje auto-regulado, motivación y cambio conceptual

Modelos didácticos

d-metacognición e intervención educativa.

Competencias: construcción de competencias

La evaluación de los aprendizajes en el contexto curricular y la evaluación formativa. Funciones de la evaluación. Importancia de la evaluación de proceso. Tipos de evaluación: diagnóstica o inicial, formativa, formadora y sumativa. Evaluación, autoevaluación, coevaluación, metaevaluación. Criterios de selección y diseño de instrumentos de evaluación.

Procesamiento de la información y análisis de sus resultados

\author{
3. Tópicos Concepción de ciencia y tecnología \\ epistemológicos Origen de algunos conceptos utilizados en química \\ Historia disciplinar en abordaje epistemológico \\ Fenómeno- modelo \\ Enfoque ciencia tecnología sociedad, estudio de casos. \\ Análisis de textos de la asignatura. \\ Educación en la diversidad. Educación en la diversidad. \\ a-diversidad en el aprendizaje. \\ b-diferencia de lenguaje en el salón de clases: dialectos y pronunciación, dialectos y \\ enseñanza, dialectos de género. \\ El currículo oculto y la evaluación.
}

Elaboración propia en base al Programa Oficial de Didáctica III de profesorado de Química - Plan 2008

Los aspectos diferenciadores de los cursos radican especialmente en el objeto de saber propio, en el conocimiento que constituye la intersección en los procesos de enseñanza y de aprendizaje de las diferentes disciplinas científicas. Por ello, los programas de los cursos de didáctica de Biología, Física y Química presentan diferencias, especialmente en los contenidos propuestos. Para el profesorado de Química el detalle de contenidos se presenta en la tabla 2. Si bien en dicho detalle se reconocen diferentes módulos o unidades con contenidos explícitos, existen contenidos transversales a la totalidad del curso. Es importante el reconocimiento de que en la especialidad química los diferentes programas de los cursos de didáctica favorecen la espiralización del conocimiento, promoviendo que a medida que el estudiante avanza en la carrera y se enfrenta cada vez con mayor grado a instancias profesionales, los contenidos se 
resignifiquen desde diversas perspectivas teóricas y problemáticas de investigación. Este último aspecto no es menor, y por ello la el curso de Didáctica III de la especialidad Química articula con el Taller del mismo nivel denominado Metodología en investigación de la Enseñanza de la Química.

Uno de los contenidos considerados transversales es la formación en la comunicación a través del lenguaje específico disciplinar, en sus diferentes niveles y sus conversiones. Para ello, además de promoverlo tanto en código verbal oral como en código escrito en distintas actividades (planificación en distintos niveles, comunicación didáctica, etc.) en el curso de Didáctica III de Química en el Ce.R.P del litoral, previamente a que se realice un análisis conjunto de las intervenciones didácticas, el estudiante responsable de dicha intervención realiza un análisis por escrito, atendiendo y promoviendo de esta manera la epistemia de la escritura (Tolchinsky Ladsmann,1993; Sutton,1997; Acevedo, 2013). Este proceso promueve también la vinculación entre los marcos teóricos de la didáctica y los hechos que emergen en la práctica profesional desde posturas reflexivas, así como también se promueven procesos metacognitivos.

Por las características descriptas, la práctica pre-profesional que se desarrolla en el Ce.R.P del Litoral, inicia e inserta en la docencia a los profesores en formación. Los inicia porque las prácticas se desarrollan en contextos educativos reales, es decir en Escuelas, planes y programas que están funcionando en la región. Es un inicio que no se hace en solitario y desvinculado del Instituto de formación. El profesor principiante tiene un "profesor adscriptor" para la práctica de $2^{\circ}$ y $3^{\circ}$ de la carrera (presente en el aula y responsable directo de la asignatura), y luego tiene un profesor tutor que los acompaña y supervisa en la práctica de $4^{\circ}$ año. También la práctica pre-profesional inserta a los profesores en formación en la docencia. En la práctica de $4^{\circ}$ año el novel docente entra en contacto con colegas del Centro de Práctica, con los cuales interacciona y coordina acciones educativas. Con estos colegas experimentados tendrá instancias de evaluación de estudiantes y participará en diferentes actividades de la Escuela: exposiciones, conmemoraciones, actos en fechas patrias, Asambleas técnico docente locales, etc.

El intercambio que se tuvo con las Universidades de Brasil, nos permitió identificar algunas diferencias con lo que sucede en nuestro Centro. De acuerdo al Plan Nacional de Educación (PNE), aprobado por el Congreso Nacional de Brasil en 2014, una de las metas prioritarias es la formación académica del profesorado (MEC/SASE, 2014). Como objetivo el 
PNE recalca la necesidad de que todos los docentes tengan un nivel superior de educación, al menos de licenciatura. Para estas carreras el tiempo general de prácticas en contextos reales, a lo largo de la carrera, es sustancialmente menor al que se tiene en Uruguay con el Plan 2008. El Consejo Nacional de Educación (CNE), establece que, de las 2800 horas totales de formación, 400 corresponden al Estágio Supervisionado, empezando las prácticas en las escuelas después de 50\% del curso, en el tercer y cuarto año de la carrera (CNE/CP No 2/2015). Las pasantías supervisadas que se desarrollan en la formación inicial de profesores en Brasil, se corresponden con las Didácticas existentes en el Plan 2008 de profesorado en Uruguay.

El desarrollo de una práctica pre-profesional con un grupo a cargo y a lo largo de todo un año lectivo, tal como se dan en la asignatura Didáctica III del Plan 2008 en Uruguay, no se tiene en ninguno de los Planes de las Universidades visitadas en Brasil. Para ellos la inserción a la docencia tal y como la presentamos en este trabajo, comienza posterior a la titulación cuando el recién egresado asume, por primera vez, un grupo de clase y desarrolla en él todas las funciones inherentes al ejercicio de la profesión. Quizá esto explica, en buena medida, lo que los colegas pasantes vienen constatando: un número importante de egresados de las carreras con formación didáctica (licenciaturas), terminan optando por no trabajar en la docencia para la cual obtuvieron un título habilitante.

Por otro lado, el contacto directo y personal entre profesores tutores de práctica del Instituto formador y los estudiantes que cursan instancias de práctica docente pre-profesional, es mayor en la experiencia de Salto. La cantidad de estudiantes matriculados en los cursos de las asignaturas en las Universidades norteñas participantes de la experiencia, sumado a las distancias geográficas existentes entre la Universidad y la Escuela donde se hace la práctica docente pre-profesional, son variables que inciden poderosamente para explicar la diferencia señalada con el Ce.R.P del Litoral. Los colegas brasileños de la pasantía expresaron al respecto que la supervisión y apoyo que se hace desde la Universidad al profesor, es fundamentalmente a través de informes de actuación que elaboran el propio profesor practicante y el profesor que los recibe en la Escuela.

Un estudio realizado en Brasil habla de fractura curricular en la formación inicial de profesores, debido en buena medida a las características que tiene la práctica docente preprofesional (Palazzo, Gomes y Rêgo Pimentel, 2016). Se señala por ejemplo que la formación inicial debería involucrar una hilatura y tejedura hacia la práctica, con cursos donde los 
estudiantes no se sientan abandonados, asustados por la incapacidad de integrar la práctica en el tejido formativo que están teniendo (Gomes, Pereira, 2009). El mencionado estudio concluye diciendo que los "estudiantes exaltaron la práctica por su valor, pero la criticaron por sus limitaciones", siendo el sentimiento que ellos experimentaron al realizarla el de "ser echados a la realidad y hacer lo que pudieran" (Palazzo, et al., 2016, p. 55 - 56).

\section{Reflexiones finales}

Iniciar en la docencia al profesor durante su formación inicial es muy significativo y relevante en el proceso de formación de identidad profesional. Se ha demostrado que las ideas asumidas durante el proceso de formación inicial, constituyen el sustrato básico de la identidad profesional del profesor, perfilando en él el primer modelo que es orientador del trabajo que va a desarrollar como profesional (Esteve, 1993).

Es en la formación docente inicial que se puede dar el primer paso hacia la inserción laboral y por ende amortiguar y soportar el primer shock con la realidad (Marcelo García, 2012). Las instituciones formadoras de profesores pueden y deben por tanto ofrecer el soporte humano y académico a los noveles docente para que el egresado no abandone la profesión y para que las

aspiraciones y objetivos de cambio e innovación educativa que se tienen en la formación inicial, no naufraguen.

La experiencia de los profesores expertos que supervisan y orientan las prácticas preprofesionales, es un recurso fundamental para iniciar en la docencia e insertar en la profesión, al profesor principiante. Es necesario que las instituciones de formación docente generen los contextos formativos para que se desarrollen procesos de reflexión, individuales y colectivo, antes de la acción docente y después de desarrollada la misma en el contexto de las escuelas de práctica (Calle García, Rodríguez Andino \& Amayuela Mora, 2015). Es necesario que los profesores en su formación inicial tengan experiencias de docencia en contextos reales, bajo la supervisión de profesores expertos que trabajan de manera sostenida el análisis crítico de los modelos de enseñanza que se dan; será esto lo que servirá de guía para las diversas etapas de la vida profesional (Vaillant, 2009). Trabajando así se conseguirá generar una relación dialéctica entre teoría y práctica.

Los contextos formativos en las instituciones de formación inicial de docentes deben favorecer la posibilidad de experimentar modelos de enseñanza diversificados en las aulas. Los 
propios formadores deben propiciar y poner a prueba distintas estrategias y recursos para enseñar en los contextos de cambios permanentes y vertiginosos que caracterizan las sociedades actuales. Como dicen Alliud y Antelo hay que probar y ensayar, sin temor, ya que los espacios de práctica pre-profesional "tienen un alto potencial formador y transformador si se convierten en ámbitos de experimentación y de puestas a prueba de experiencias pedagógicas diversas" (Alliud y Antelo, 2009, p. 97).

Lo referido en el presente documento es resultado del trabajo colaborativo y reflexivo entre docentes de didáctica que construyen a diario parte de la formación profesional de futuros educadores. Trabajo que se realiza en comunidad de aprendizaje, significando y resignificando la didáctica y la práctica docente desde colectivos docentes, equipos de gestión de las instituciones involucradas, integrando a los estudiantes y buscando construir redes con otras instituciones de formación docente del país y la región. El camino de la formación en educación abre día a día variados desafíos, quizás uno de los más urgentes a atender sea la incorporación de la investigación fuertemente unida a la didáctica y a la práctica docente.

\section{Referencias bibliográficas}

Acevedo, C. (2013). La función epistémica de la escritura para aprender ciencias naturales en el contexto escolar. Legenda, 17 (16), 44-68. Recuperado de: http://erevistas.saber.ula.ve/index.php/legenda/article/view/4580

Alliaud, A. y Antelo, E. (2009). Iniciarse a la docencia. Los gajes del oficio de enseñar. Revista Profesorado, 13 (1), 89-100. Recuperado de: https://www.ugr.es/ recfpro/rev131ART6.pdf

Arias Regalía, D., Bonan, L. y Gonçalves, P. (2016). Acciones de formación docente para la enseñanza de las ciencias de la Tierra. Actas del XIX Simposio de Enseñanza de las Ciencias de la Tierra (Manresa, España): 43-54. Recuperado de: https://www.academia.edu/40851711/ACCIONES_DE_FORMACI\%C3\%93N_DOCE NTE_PARA_LA_ENSE\%C3\%91ANZA_DE_LAS_CIENCIAS_DE_LA_TIERRA

Branda, S. (2015). Voces y relatos de iniciación: las prácticas pre-profesionales y su rol en la construcción del perfil profesional de los alumnos del profesorado. VIII Jornadas Nacionales y $1^{\mathrm{o}}$ Congreso Internacional sobre la Formación del Profesorado. Recuperado

de: 
https://www.academia.edu/18040360/VOCES_Y_RELATOS_DE_INICIACI\%C3\%93 N_LAS PR $\%$ C $3 \% 81 C T I C A S$ PRE-

PROFESIONALES Y SU ROL EN LA CONSTRUCCI\%C3\%93N DEL PERFIL PROFESIONAL_DE_LOS_ALUMNOS DEL_PROFESORADO

Bullough, R.V. (2000). Convertirse en profesor: la persona y la localización social de la formación del profesorado. En Biddle, B.J.; Good, T.L; Gooddson, I.F. La enseñanza y los profesores I (La profesión de enseñar). Barcelona: Paidós, 99-166.

Calle García, R.; Rodríguez Andino, M. y Amayuela Mora, G. (2015). Los procesos de reflexión colectiva durante la práctica pre-profesional en la formación de profesores en Ecuador. Transformación, 11 (1), 82-91.

CNE (2015). Diretrizes Curriculares Nacionais para a formação inicial em nível superior e para a formação continuada. Brasil. Recuperado de: http://portal.mec.gov.br/index.php?option=com_docman\&view=download\&alias $=176$ 25-parecer-cne-cp-2-2015-aprovado-9-junho-2015\&category slug=junho-2015pdf\&Itemid=30192

Cochran-Smith, M. y Fries, K. (2005). Studying teacher education. The report of the AERA Panet of Research and Teacher Education, Lawrence Erlbaum, New Jersey

Correa, E.; Cividini, M. y Fuentealba, R. (2013). Formación inicial e inserción profesional: procesos Clave para la profesionalización docente. En: Correa, E., Cividini, M., Fuentealba, R. y Boerr, I. (Coord.). Formación e Inserción Profesional: Desafios y pistas para la Profesionalización Docente. Santiago: Metas Educativas, p. 9-18, 2013.

Day, C. (2005). Formar docentes. Cómo, cuándo y en qué condiciones aprende el profesorado. Madrid: Narcea.

Esteve, J. (1993). El choque de los principiantes con la realidad. Cuadernos de Pedagogía, 220, 58-63. Recuperado

de: https://www.academia.edu/20801180/El_choque_de_los_principiantes_con_la_realida d

Fernández, G. y Clot, Y. (2010). Entrevistas en auto-confrontación: un método en clínica de la actividad. Informática na educacao: teoria y prática, 13 (1), 11-16. Recuperado de: http://seer.ufrgs.br/index.php/InfEducTeoriaPratica/article/view/15135/12048

Freire, P. (1997). Pedagogía de la autonomía. Buenos Aires: Siglo XXI Editores.

Gomes, C. y Pereira, M. (2009). A formação do professor em face das violências das/nas escolas. Cadernos de Pesquisa, 39 (136), 201-224.

Grilli Silva, J. y Silva Casterá, L. (2015). Análisis colectivo de las prácticas de aula. Dispositivos en la formación inicial de profesores que favorecen el aprendizaje colaborativo. Revista Electrónica Diálogos Educativos, 15, 69-89. Recuperado de: http://revistas.umce.cl/index.php/dialogoseducativos/article/view/1020

Imbernón, F. (2007). La formación permanente del profesorado. Nuevas ideas para formar en la innovación y el cambio. Barcelona: Graó.

Marcelo García, C. (coord.) (2008). El profesorado principiante. Inserción a la docencia. Octaedro, Barcelona 
Marcelo García, C. (2012). Empezar con buen pie: inserción a la enseñanza para profesores principiantes. Olhar de professor, Ponta Grossa, 15(2): 209-221. Recuperado de: https://pdfs.semanticscholar.org/c591/3e64698d1b9e4ca38e39c5d6056fb321a520.pdf

MEC/SASE (2014). Planejando a próxima década: conhecendo as 20 metas do Plano Nacional de Educação. Recuperado de: http://pne.mec.gov.br/images/pdf/pne_conhecendo_20_metas.pdf

McKinsey, C. (2007). How the World's Best Performing School Systems Come Out on Top. Recuperado de: http://www.mckinsey.com/clientservice/socialsector/resources/pdf/Worlds_School_Sy stems Final.pdf

Meinardi, E.; Regalía, D. y Plaza, V. (2018). Propuestas didácticas para enseñar Ciencias Naturales y Matemática VI. Ciudad Autónoma de Buenos Aires: Ediciones Fundalma, 2018. Libro digital, PDF. Recuperado de: http://www.ccpems.exactas.uba.ar/cms/index.php/producciones-didacticas/599propuestas-didacticas-para-ensenar-ciencias-naturales-y-matematica-vi-2018

OCDE (2005). Teachers matter: attracting, developing and retaining effective teachers, OCDE, Paris.

Palazzo, J., Gomes, C. y Rêgo Pimentel, G. (2016). La práctica en la formación de educadores en Brasil: currículos fracturados. Revista Electrónica Interuniversitaria de Formación del Profesorado, 19(2), 45-58.

Perrenoud, P. (2004). Desarrollar la práctica reflexiva en el oficio de enseñar. Profesionalización y razón pedagógica. Barcelona: Graó.

Pesce, F. (2014). La didáctica en la formación de docentes para la enseñanza media en Uruguay. Intercambios, 1 (1), 53-61. Recuperado de: https://ojs.intercambios.cse.udelar.edu.uy/index.php/ic/article/view/12

Rickenmann, R. (2007). Investigación y formación docente: dispositivos de formación y elementos para la construcción de una identidad profesional. EccoS-Revista Cientifica, $\begin{array}{lllll}\text { São } & \text { Paulo, } & 9 & \text { (2), 435-463. Recuperado de: }\end{array}$ http://www.uninove.br/PDFs/Publicacoes/eccos/eccos_v9n2/eccosv9n2_2i14.pdf

Rodríguez Zidán, E.; Grilli Silva, J. y Rodríguez, S. (2020). Formación de profesores en Uruguay. Tensiones y desafíos en el proceso de transición hacia un modelo universitario de la formación docente. Revista Brasileira de Pesquisa sobre Formação de Professores, 12 (23), 99-114 Recuperado de: http://repositorio.cfe.edu.uy/handle/123456789/742

Rodríguez, M., Calle, R. y Zabala, S. (2018). Modelo para desarrollar prácticas preprofesionales reflexivas en la formación de docentes para educación básica. Revista Espacios, $39 \quad$ (46), 22-27. Recuperado de: http://www.revistaespacios.com/a18v39n46/a18v39n46p22.pdf

Schön, D. A. (1992). La formación de profesionales reflexivos. Hacia un nuevo diseño de la enseñanza y el aprendizaje en las profesiones. Barcelona: Paidós.

Sutton, C. (1997). Ideas sobre la ciencia e ideas sobre el lenguaje. Alambique, 12, 8-32

Tardif, M. (2004). Los Saberes del Docente y su Desarrollo Profesional. Madrid: Narcea. 
Tardif, M. y Borges, C. (2013). La inserción profesional de docentes nóveles: tendencias recientes y retos futuros. En: Correa, E., Cividini, M., Fuentealba, R. y Boerr, I. (Coord.). Formación e Inserción Profesional: Desafios y pistas para la Profesionalización Docente. Santiago: Metas Educativas, 2013, 19-44.

Terigi, F. (2007). Desarrollo profesional continuo y carrera docente en América Latina PREAL Serie Documentos $\quad \mathrm{N}^{\mathrm{o}} 50 . \quad$ Recuperado de: https://grupodis.net/images/docs_gestrategica/PREAL_Desarrollo $\% 20$ Profesional $\% 20$ Coontinuo\%20y\%20Carrera\%20docente\%20en\%20Ame\%CC\%81rica\%20Latina 201 $\underline{0 . p d f}$

Tolchinsky Ladsmann, L. (1993) Los procesos de producción y calidad de los textos, en Aprendizaje del lenguaje escrito: procesos evolutivos e implicaciones didácticas. Barcelona: Anthropos.

Vaillant, D. (2009). Políticas de inserción a la docencia en América Latina: la deuda pendiente. Profesorado, Revista del currículum y formación del profesorado, 13 (1), 27-41. Recuperado de: https://www.ugr.es/ recfpro/rev131ART2.pdf

Vaillant, D. (2013). Formación inicial del profesorado en América Latina: Dilemas centrales y perspectivas. Revista Española de Educación Comparada, 22, 185-206. Recuperado de: http://revistas.uned.es/index.php/REEC/article/view/9329

Vaillant, D. y Marcelo García, C. (2015). El A, B, C, D de la Formación Docente. Madrid: Narcea.

Vonk, J. (1996). A Knowledge Base for Mentors of Beginning Teachers: Results of a Dutch Experience. In R. McBridge (Ed.), Teacher Education Policy, (p. 112-134). London: Falmer Press.

Zabalza, M. (2009). Prácticum y formación: ¿En qué puede formar el prácticum? En Raposo, M., Martínez, M., Lodeiro, L., Fernández de la Iglesia, J. y Pérez, A. (Coords.). In Pontevedra (Ed.), El Prácticum más allá del empleo: Formación vs. Training (pp. 45 $\square$ 65).

Zeichner, K. M. (1995). Los profesores como profesionales reflexivos y la democratización de la reforma escolar. En Pérez, A. (1995), Volver a pensar la educación (Vol. II, págs. 385-398). Madrid: Morata-Paideia. 
Políticas, Programas y Prácticas de Formación de Profesores

i https://www.capes.gov.br/images/stories/download/editais/resultados/26122017-RESULTADOCONVOCATORIA-DE-PASANTIAS-MERCOSUL.pdf 